\title{
Ages and chemical compositions of massive clusters in NGC147 and M31
}

\author{
Margarita Sharina ${ }^{1}$ and Vladislav Shimansky ${ }^{2}$ \\ ${ }^{1}$ Special Astrophysical Observatory, Russian Academy of Sciences, Nizhnii Arkhyz, 369167 \\ Russia \\ ${ }^{2}$ Kazan (Volga region) Federal university, Kazan, 420008 Russia \\ email: sme@sao.ru, Slava.Shimansky@kpfu.ru
}

\begin{abstract}
We present estimates of ages, $[\mathrm{Fe} / \mathrm{H}]$, helium content $(\mathrm{Y})$ and abundances of $\mathrm{C}, \mathrm{N}$, $\mathrm{Mg}, \mathrm{Ca}$, and several other elements for the following globular clusters (GCs): GC7 in NGC147, and Mayall II, Mackey 1 and Mackey 6 in M31. Medium-resolution integrated-light spectra of the GCs were conducted with the $6 \mathrm{~m}$ telescope. To derive the ages and abundances for the GCs we carried out their population synthesis using model stellar atmospheres, the Padova YZVAR isochrones and the Chabrier mass function. We compare the results with the corresponding data obtained using the same method for several massive Galactic GCs. We show that the differences in the light-element abundances between GCs with similar ages and metallicities may reach 0.5-0.6 dex. The corresponding differences for other elements are usually 2-3 times smaller. We suggest that at least partially the detected differences may be due to light-element abundance variations in the atmospheres of high-luminosity red giant branch stars as a consequence of the transportation of the produced elements to the surface layers.
\end{abstract}

Keywords. galaxies: individual (M31, NGC147) - galaxies: star clusters: abundances

Old massive GCs in different galaxies show similar structural properties and photometric colors (e.g. Veljanoski et al. /, 2013). It is not known whether the processes of their formation were also similar and whether element abundance variations exist in most massive of them as it is observed in our Galaxy (Gratton et al. /, 2012).

We carry out population synthesis of medium-resolution (FWHM $\sim 5 \AA$ ) integrated light spectra of massive GCs near M31 using model stellar atmospheres and estimate abundances of chemical elements. The observational instrumental setup and methods of the spectroscopic reduction are described by Makarov et al. (2015). Our method of chemical element abundance analysis and determination of evolutionary parameters using stellar atmosphere models was described by Sharina et al. (2014, 2013, hereafter: S14, 13) and Khamidullina et al. (2014a,b, hereafter: Kh14a, b). In these papers the method was tested using medium-resolution integrated light spectra of Galactic GCs. In particular, it was shown by S13 that it is possible to derive simultaneously age and helium abundance using medium-resolution integrated light spectra of GCs, because of the influence of these two parameters on the hydrogen absorption line profiles.

Table 1. Age [Gyr], metallicity [dex] and helium abundance [dex] for the studied GCs.

\begin{tabular}{llll|ll}
\hline Object & $\log \mathrm{t}($ ours $)$ & {$[\mathrm{Fe} / \mathrm{H}]$ (ours) } & $Y$ (ours) & $\log \mathrm{t}(\mathrm{Lit})$. & {$[\mathrm{Fe} / \mathrm{H}]$ (Lit.) } \\
\hline GC7-N 147 & $10.1 \pm 0.1$ & $-1.70 \pm 0.1$ & $0.30 \pm 0.03$ & $9.9 \pm 0.12^{7}$ & $-1.5 \pm 0.2^{7},-1.8 \pm 0.3^{8}$ \\
Mayall-II & $10.15 \pm 0.05$ & $-1.00 \pm 0.05$ & $0.26 \pm 0.02$ & $10.176^{6}, 10.26 \pm 0.4^{4}$ & $-0.95 \pm 0.09^{6},-1.08 \pm 0.09^{3},-0.9^{2}$ \\
MGC 1 & $10.0 \pm 0.05$ & $-2.20 \pm 0.1$ & $0.30 \pm 0.03$ & $9.9 \pm 0.1^{4}$ & $-2.14^{5},-2.15^{2}$ \\
MGC 6 & $10.0 \pm 0.1$ & $-1.85 \pm 0.1$ & $0.30 \pm 0.03$ & $10.3 \pm 0.1^{4}, 10.134^{1}$ & $-2.07 \pm 0.18^{1},-2.14^{5},-1.8^{2}$ \\
\hline
\end{tabular}

Notes: 1: Fan et al. (2012); 2: Federici et al. (2012); 3:Huchra et al. (1991); 4: Ma (2012); 5: Mackey et al. (2007); 6: Meylan et al. (2001); 7: Sharina \& Davoust (2009); 8: Veljanoski et al. (2013) 

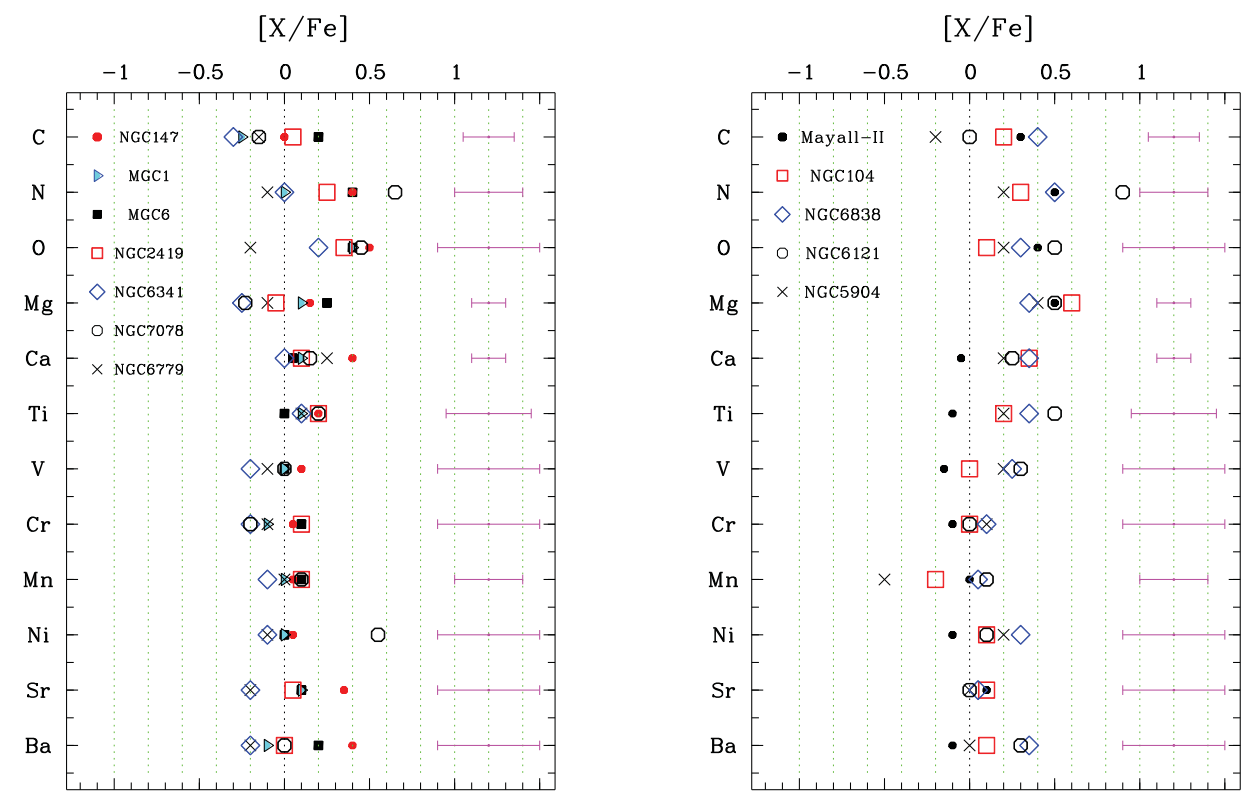

Figure 1. Comparison of chemical abundances derived using our method for GC7-NGC147, MGC1, MGC6, Mayall-II and for several Galactic GCs. Left: $[\mathrm{Fe} / \mathrm{H}] \sim-2.0$ dex; right: $\sim-1.0$ dex. Errors of abundance determination are shown in the right parts of the two plots.

Our results of age, metallicity and helium abundance determination and data from the literature are listed in Table 1. Comparison of chemical abundances for the four extragalactic GCs with the corresponding data for Galactic GCs of similar metallicity derived using the same method (S14, 13; Kh14a, b) is shown in Fig. 1. It is seen that the differences in the light-element abundances between individual GCs in the low-metallicity group (left panel of Fig. 1) and in the high-metallicity group (right panel) are on average larger than the corresponding differences in the abundances of other elements.

Acknowledgements. This presentation was supported by the Russian Scientific Foundation grant 14-12-00965.

\section{References}

Fan, Zh., Huang, Y.-F., Li, J.-Z., Zhou, Xu, Ma, Jun, \& Zhao, Y.-H. 2012, Research in Astronomy and Astrophysics, 12, 829

Federici, L., Cacciari, C., Bellazzini, M., Fusi, Pecci F., Galleti, S., \& Perina, S 2012, A\&A, 544, 155

Gratton, R. G., Carretta, E., \& Bragaglia A. $2012 A \& A R, 20,50$

Huchra, J. P., Brodie, J. P., \& Kent, S. M. 1991, ApJ, 370, 495

Khamidullina, D., Sharina, M., Shimansky, V., \& Davoust, E. 2014a, Astrophysical Bulletin, 69, 409

Khamidullina, D., Sharina, M., Shimansky, V., \& Davoust, E. 2014b, Baltik Astronomy, 23, 260

Ma, Jun 2012, Research in Astronomy and Astrophysics, 12, 115

Mackey, A. D. et al. 2007, ApJ (Letters), 655, 85

Makarov, D. I., Sharina, M. E., Karachentseva, V. E., \& Karachentsev, I. D. $2015 A \& A, 581,82$

Meylan, G., Sarajedini, A., Jablonka, P., Djorgovski, S., Bridges, T., \& Rich, R. 2001, AJ, 122, 830

Sharina, M., Donzelli, C., Davoust, E., Shimansky, V., \& Charbonnel, C. 2014, A\&A, 570, 48

Sharina, M. E., Shimansky, V., \& Davoust, E. 2013, Astronomy Reports, 57, 410

Sharina, M. E., \& Davoust, E. 2009, A\&A, 497, 65

Veljanoski, J. et al. 2013, MNRAS, 435, 3654 